\title{
Review of: "Multiple spillovers and onward transmission of SARS-Cov-2 in free-living and captive White-tailed deer (Odocoileus virginianus)"
}

Jan Novakofski ${ }^{1}$

1 University of Illinois at Urbana-Champaign

Potential competing interests: The author(s) declared that no potential competing interests exist.

A potentially quite fascinating paper but with many issues that must be addressed before it is acceptable for publication.

Observations without analysis are not "strong evidence" but just observations. For example, "strong evidence of temporal clustering" is not accompanied by a temporal or spatial cluster analysis. Similarly, "strong evidence of multiple likely zooanthroponotic spillovers" (while it seems rather likely) is not accompanied by any analysis.

Conversely, important questions about zoonotic mechanism where speculation would be useful are not discussed. For example. given what we know about COVID transmission (the virus does not remain infectious very long in the environment) it is likely that 1) Infected workers on captive deer facilities infected captive deer, which subsequently escaped and transmitted virus to wild deer or 2) people put out infected bait before hunting season that infected wild deer directly. This second path seems reasonable given the statistically lower incidence in captive deer compared to wild deer.

The manuscript could benefit from error checking. For example, the monthly positive case \#s in figure 2 add up to more than the total cases in the summary map panel. Terminology should be more consistent with current literature. For example, supplemental tables refer to captive deer, meaning deer separated by fences from wild deer, while the manuscript refers to these deer as coming from a game preserve, which is an area of land in which hunting is strictly controlled but not fenced so deer would not separated from other wild deer. The distinction has important implications. Similarly, "reservoir" has a rather specific meaning and more careful use of this term until there is evidence would be appropriate.

Given that these problems can be fixed easily, the manuscript is interesting and I would encourage corrections and full submission. 